\title{
Hipoxemia crónica asintomática en contexto de malformaciones arteriovenosas pulmonares: Síndrome de Rendu-Osler-Weber
}

\author{
Chronic asymptomatic hypoxemia in the context of a pulmonary arteriovenous \\ malformations: Rendu-Osler-Weber syndrome
}

\author{
Diego Carvallo-Claros, Evelyn A. Fiori y Luis G. García-Chamorro* \\ Servicio de Cardiología, Sanatorio Güemes, Buenos Aires, Argentina
}

Más del $70 \%$ de las malformaciones arteriovenosas pulmonares (MAVP) son congénitas y están relacionadas con el síndrome hereditario de telangiectasias hemorrágicas $(\mathrm{HHT})$, también conocido como síndrome de Rendu-Osler-Weber ${ }^{1}$.

Presentamos el caso de un paciente de sexo masculino de 23 años en clase funcional I, con antecedentes de epistaxis recurrente y madre con MAV cerebral, quien consultó por contacto con un caso positivo para enfermedad por coronavirus-2019 (COVID-19). Durante la valoración se constató oximetría del $89 \%$ y acropaquia, sin alteraciones en el examen físico cardiopulmonar y electrocardiograma normal, la radiografía de tórax evidenció imagen radiopaca bien definida en campo pulmonar medio derecho (Fig. 1A), el laboratorio reportó poliglobulia y reacción en cadena de la polimerasa negativa para COVID-19. La tomografía de tórax mostró MAVP en segmento posterior del lóbulo superior derecho y medial del lóbulo medio (Fig. 1 B y C), realizándose el diagnóstico de HHT por criterios de Curaçao. En el ecocardiograma transesofágico se evidenció pasaje de burbujas a cavidades izquierdas en el tercer latido sin observarse solución de continuidad en el septum interventricular (SIV), ni dilatación de cavidades. La angiografía pulmonar confirmó la existencia de dos

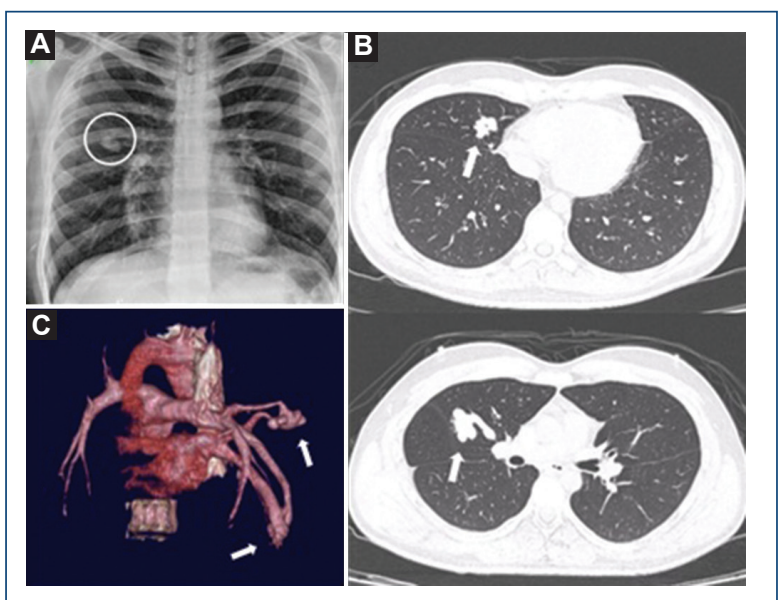

Figura 1. Radiografía de tórax.

MAVP de $10 \mathrm{~mm}$ a nivel de la arteria pulmonar derecha con drenajes en la vena pulmonar superior y a nivel del ramo lobar medio (Fig. 2A) con presiones sistólica, diastólica y media de la arteria pulmonar de 20, 13 y $15 \mathrm{mmHg}$ respectivamente. Se realizó la embolización percutánea con implante de un plug Cardi-O-fix ${ }^{\circledR}$ occluder system de 14 x $10 \mathrm{~mm}$ en cada rama (Fig. 2 B y C). Durante el procedimiento se evidenció una tercera

\section{Correspondencia:}

*Luis Guillermo García Chamorro

E-mail: luguigacha@gmail.com
Disponible en internet: 16-11-2021 Arch Cardiol Mex. (ahead of print) www.archivoscardiologia.com 1405-9940 / @ 2021 Instituto Nacional de Cardiología Ignacio Chávez. Publicado por Permanyer. Este es un artículo open access bajo la licencia CC BY-NC-ND (http://creativecommons.org/licenses/by-nc-nd/4.0/). 

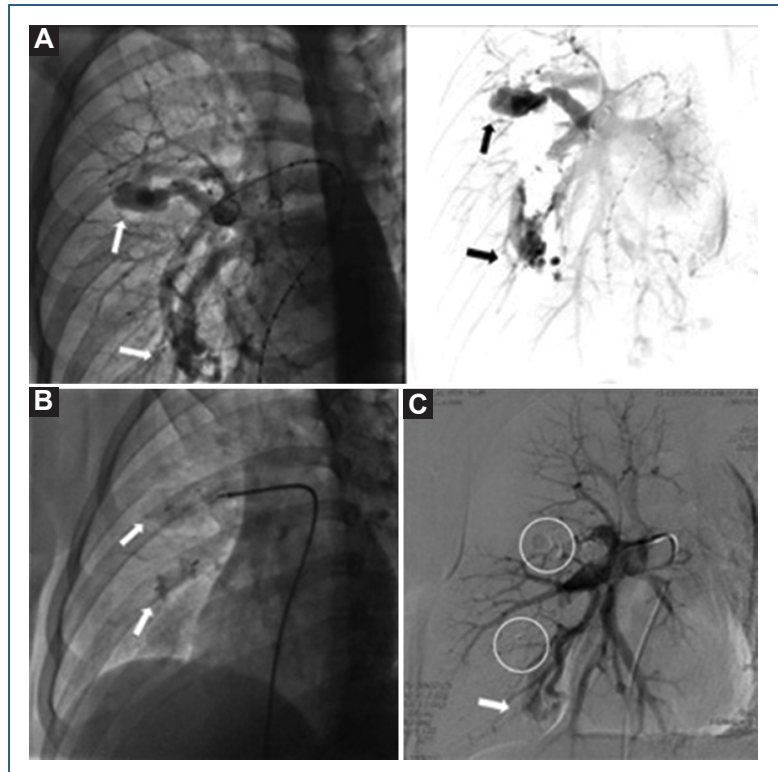

Figura 2. Angiografía pulmonar.

MAVP a nivel basal, cerrándose en un segundo tiempo (Fig. 2C). Ambos procedimientos fueron bien tolerados, con mejoría inmediata de la saturación (96\%).

Como reportamos en nuestro caso, el manejo endovascular de las MAVP es el tratamiento estándar²; aunque en la literatura la mayoría de las lesiones tratadas por esta vía son únicas, se ha reportado la oclusión de hasta cinco lesiones en un mismo pulmón de manera excepcional ${ }^{3}$.

\section{Financiamiento}

La presente investigación no ha recibido ninguna beca específica de agencias de los sectores públicos, comercial o sin ánimo de lucro.

\section{Conflicto de intereses}

Los autores declaran no tener conflicto de intereses.

\section{Responsabilidades éticas}

Protección de personas y animales. Los autores declaran que para esta investigación no se han realizado experimentos en seres humanos ni en animales.

Confidencialidad de los datos. Los autores declaran que han seguido los protocolos de su centro de trabajo sobre la publicación de datos de pacientes.

Derecho a la privacidad y consentimiento informado. Los autores han obtenido el consentimiento informado de los pacientes y/o sujetos referidos en el artículo.

\section{Bibliografía}

1. Majumdar S, McWilliams JP. Approach to pulmonary arteriovenous malformations: A comprehensive update. J Clin Med. 2020;9(6):1927.

2. Meier NM, Foster ML, Battaile JT. Hereditary hemorrhagic telangiectasia and pulmonary arteriovenous malformations: clinical aspects. Cardiovasc Diagn Ther. 2018;8(3):316-24.

3. Ruiz-Ontiveros MA, Flores-Arizmendi A, Salgado-Sandoval A. Percutaneous occlusion of five pulmonary arteriovenous fistulas in the same pulmonary lobe. Arch Cardiol Mex. 2021 Sep 7. doi: 10.24875/ACM.21000066. Epub ahead of print. 